\title{
Explicit averaging cyclic algorithm for common fixed points of a finite family of asymptotically strictly pseudocontractive mappings in $q$-uniformly smooth Banach spaces
}

Ying Zhang ${ }^{1,2^{*}}$ and Zhiwei Xie ${ }^{3}$

\author{
"Correspondence: \\ spzhangying@126.com \\ 'School of Mathematics and \\ Physics, North China Electric Power \\ University, Baoding, Hebei 071003, \\ P.R. China \\ ${ }^{2}$ School of Economics, Renmin \\ University of China, Beijing, 100872, \\ P.R. China \\ Full list of author information is \\ available at the end of the article
}

\begin{abstract}
Let $E$ be a real $q$-uniformly smooth Banach space which is also uniformly convex and $K$ be a nonempty, closed and convex subset of $E$. We obtain a weak convergence theorem of the explicit averaging cyclic algorithm for a finite family of asymptotically strictly pseudocontractive mappings of $K$ under suitable control conditions, and elicit a necessary and sufficient condition that guarantees strong convergence of an explicit averaging cyclic process to a common fixed point of a finite family of asymptotically strictly pseudocontractive mappings in q-uniformly smooth Banach spaces. The results of this paper are interesting extensions of those known results. MSC: $47 \mathrm{H} 09 ; 47 \mathrm{H} 10$
\end{abstract}

Keywords: asymptotically strictly pseudocontractive mappings; weak and strong convergence; explicit averaging cyclic algorithm; fixed points; q-uniformly smooth Banach spaces

\section{Introduction}

Let $E$ and $E^{*}$ be a real Banach space and the dual space of $E$, respectively. Let $J_{q}(q>1)$ denote the generalized duality mapping from $E$ into $2^{E^{*}}$ given by $J_{q}(x)=\left\{f \in E^{*}:\langle x, f\rangle=\right.$ $\|x\|^{q}$ and $\left.\|f\|=\|x\|^{q-1}\right\}$ for all $x \in E$, where $\langle\cdot, \cdot\rangle$ denotes the generalized duality pairing between $E$ and $E^{\prime \prime}$. In particular, $J_{2}$ is called the normalized duality mapping and it is usually denoted by $J$. If $E$ is smooth or $E^{*}$ is strictly convex, then $J_{q}$ is single-valued. In the sequel, we will denote the single-valued generalized duality mapping by $j_{q}$.

Let $K$ be a nonempty subset of $E$. A mapping $T: K \rightarrow K$ is called asymptotically $\kappa$-strictly pseudocontractive with sequence $\left\{\kappa_{n}\right\}_{n=1}^{\infty} \subseteq[1, \infty)$ such that $\lim _{n \rightarrow \infty} \kappa_{n}=1$ (see, e.g., $[1-3])$ if for all $x, y \in K$, there exist a constant $\kappa \in[0,1)$ and $j_{q}(x-y) \in J_{q}(x-y)$ such that

$$
\left\langle T^{n} x-T^{n} y, j_{q}(x-y)\right\rangle \leq \kappa_{n}\|x-y\|^{q}-\kappa\left\|x-y-\left(T^{n} x-T^{n} y\right)\right\|^{q}, \quad \forall n \geq 1 .
$$


If $I$ denotes the identity operator, then (1) can be written in the form

$$
\begin{aligned}
& \left\langle\left(I-T^{n}\right) x-\left(I-T^{n}\right) y, j_{q}(x-y)\right\rangle \\
& \quad \geq \kappa\left\|\left(I-T^{n}\right) x-\left(I-T^{n}\right) y\right\|^{q}-\left(\kappa_{n}-1\right)\|x-y\|^{q} .
\end{aligned}
$$

The class of asymptotically $\kappa$-strictly pseudocontractive mappings was first introduced in Hilbert spaces by Qihou [3]. In Hilbert spaces, $j_{q}$ is the identity, and it is shown by Osilike et al. [2] that (1) (and hence (2)) is equivalent to the inequality

$$
\left\|T^{n} x-T^{n} y\right\|^{2} \leq \lambda_{n}\|x-y\|^{2}+\lambda\left\|x-y-\left(T^{n} x-T^{n} y\right)\right\|^{2},
$$

where $\lim _{n \rightarrow \infty} \lambda_{n}=\lim _{n \rightarrow \infty}\left[1+2\left(\kappa_{n}-1\right)\right]=1, \lambda=(1-2 \kappa) \in[0,1)$.

A mapping $T$ with domain $D(T)$ and range $R(T)$ in $E$ is called strictly pseudocontractive of Browder-Petryshyn type [4] if for all $x, y \in D(T)$, there exist $\kappa \in[0,1)$ and $j_{q}(x-y) \in$ $J_{q}(x-y)$ such that

$$
\left\langle T x-T y, j_{q}(x-y)\right\rangle \leq\|x-y\|^{q}-\kappa\|x-y-(T x-T y)\|^{q} .
$$

If $I$ denotes the identity operator, then (3) can be written in the form

$$
\left\langle(I-T) x-(I-T) y, j_{q}(x-y)\right\rangle \geq \kappa\|(I-T) x-(I-T) y\|^{q} .
$$

In Hilbert spaces, (3) (and hence (4)) is equivalent to the inequality

$$
\|T x-T y\|^{2} \leq\|x-y\|^{2}+k\|x-y-(T x-T y)\|^{2}, \quad k=(1-2 \kappa)<1 .
$$

It is shown in [5] that the class of asymptotically $\kappa$-strictly pseudocontractive mappings and the class of $\kappa$-strictly pseudocontractive mappings are independent.

A mapping $T$ is said to be uniformly L-Lipschitzian if there exists a constant $L>0$ such that, for all $x, y \in K$,

$$
\left\|T^{n} x-T^{n} y\right\| \leq L\|x-y\|, \quad n \geq 1 .
$$

Let $\left\{T_{j}\right\}_{j=0}^{N-1}$ be $N$ asymptotically strictly pseudocontractive self-mappings of $K$, and denote the common fixed points set of $\left\{T_{j}\right\}_{j=0}^{N-1}$ by $F:=\bigcap_{j=0}^{N-1} F\left(T_{j}\right)$, where $F\left(T_{j}\right):=\{x \in K$ : $\left.T_{j} x=x\right\}$. We consider the following explicit averaging cyclic algorithm.

For a given $x_{0} \in K$, and a real sequence $\left\{\alpha_{n}\right\}_{n=0}^{\infty} \subseteq(0,1)$, the sequence $\left\{x_{n}\right\}_{n=0}^{\infty}$ is generated as follows:

$$
\begin{aligned}
& x_{1}=\alpha_{0} x_{0}+\left(1-\alpha_{0}\right) T_{0} x_{0}, \\
& x_{2}=\alpha_{1} x_{1}+\left(1-\alpha_{1}\right) T_{1} x_{1}, \\
& \vdots \\
& x_{N}=\alpha_{N-1} x_{N-1}+\left(1-\alpha_{N-1}\right) T_{N-1} x_{N-1}, \\
& x_{N+1}=\alpha_{N} x_{N}+\left(1-\alpha_{N}\right) T_{0}^{2} x_{N},
\end{aligned}
$$




$$
\begin{aligned}
& x_{N+2}=\alpha_{N+1} x_{N+1}+\left(1-\alpha_{N+1}\right) T_{1}^{2} x_{N+1}, \\
& \vdots \\
& x_{2 N}=\alpha_{2 N-1} x_{2 N-1}+\left(1-\alpha_{2 N-1}\right) T_{N-1}^{2} x_{2 N-1}, \\
& x_{2 N+1}=\alpha_{2 N} x_{2 N}+\left(1-\alpha_{2 N}\right) T_{0}^{3} x_{2 N}, \\
& x_{2 N+2}=\alpha_{2 N+1} x_{2 N+1}+\left(1-\alpha_{2 N+1}\right) T_{1}^{3} x_{2 N+1},
\end{aligned}
$$

The algorithm can be expressed in a compact form as

$$
x_{n+1}=\alpha_{n} x_{n}+\left(1-\alpha_{n}\right) T_{i(n)}^{k(n)} x_{n}, \quad n \geq 0,
$$

where $n=(k-1) N+i$ with $i=i(n) \in I=\{0,1,2, \ldots, N-1\}, k=k(n) \geq 1$ a positive integer and $\lim _{n \rightarrow \infty} k(n)=\infty$. The cyclic algorithm was first studied by Acedo and Xu [6] for the iterative approximation of common fixed points of a finite family of strictly pseudocontractive mappings in Hilbert spaces, and it is better than implicit iteration methods.

In [7] Xiaolong Qin et al. proved the following theorem in a Hilbert space.

Theorem QCKS Let $K$ be a closed and convex subset of a Hilbert space $H$ and $N \geq 1$ be an integer. Let, for each $1 \leq i \leq N, T_{i}: K \rightarrow K$ be an asymptotically $\kappa_{i}$-strictly pseudocontractive mapping for some $0 \leq \kappa_{i}<1$ and a sequence $\left\{k_{n, i}\right\}$ such that $\sum_{n=0}^{\infty}\left(k_{n, i}-1\right)<$ $\infty$. Let $\kappa=\max \left\{\kappa_{i}: 1 \leq i \leq N\right\}$ and $\kappa_{n}=\max \left\{\kappa_{n, i}: 1 \leq i \leq N\right\}$. Assume that $F \neq \emptyset$. For any $x_{0} \in K$, let $\left\{x_{n}\right\}$ be the sequence generated by the cyclic algorithm (5). Assume that the control sequence $\left\{\alpha_{n}\right\}$ is chosen such that $\kappa+\epsilon \leq \alpha_{n} \leq 1-\epsilon$ for all $n \geq 0$ and a small enough constant $\epsilon \in(0,1)$. Then $\left\{x_{n}\right\}$ converges weakly to a common fixed point of the family $\left\{T_{i}\right\}_{i=1}^{N}$.

Osilike and Shehu [8] extended the result of Theorem QCKS from a Hilbert space to 2-uniformly smooth Banach spaces which are also uniformly convex. They proved the following theorem.

Theorem OS Let E be a real 2-uniformly smooth Banach space which is also uniformly convex, and $K$ be a nonempty, closed and convex subset of $E$. Let $\left\{T_{j}\right\}_{j=0}^{N-1}$ be $N$ asymptotically $\lambda_{j}$-strictly pseudocontractive self-mappings of $K$ for some $0 \leq \lambda_{j}<1$ with a sequence $\left\{\kappa_{n}^{(j)}\right\}_{n=0}^{\infty} \subset[1, \infty)$ such that $\sum_{n=0}^{\infty}\left(\kappa_{n}^{(j)}-1\right)<\infty, \forall j \in J=\{0,1,2, \ldots, N-1\}$, and $F \neq \emptyset$. Let $\left\{\alpha_{n}\right\}$ satisfy the conditions

$$
\begin{aligned}
& \text { (i") } \quad 0 \leq \alpha_{n}<1, \quad n \geq 0, \\
& \text { (ii") } \quad 0<a \leq 1-\alpha_{n} \leq b<\frac{2 \lambda}{C_{2}},
\end{aligned}
$$

where $\lambda=\min _{j \in J}\left\{\lambda_{j}\right\}$ and $C_{2}$ is the constant appearing in the inequality (7) with $q=2$. Let $\left\{x_{n}\right\}$ be the sequence generated by the cyclic algorithm (5). Then $\left\{x_{n}\right\}$ converges weakly to a common fixed point of the family $\left\{T_{j}\right\}_{j=0}^{N-1}$.

We would like to point out that the condition (ii ${ }^{*}$ ) in Theorem OS excludes the natural choice $1-\frac{1}{n}$ for $\alpha_{n}$. This is overcome by this paper. Moreover, we improve and extend the 
result of Theorem OS from 2-uniformly smooth Banach spaces to $q$-uniformly smooth Banach spaces which are also uniformly convex. We prove that if $\left\{\alpha_{n}\right\}$ satisfies the conditions

(i) $\quad \mu \leq \alpha_{n}<1, \quad n \geq 0$,

(ii) $\sum_{n=0}^{\infty}\left(1-\alpha_{n}\right)\left[q \lambda-C_{q}\left(1-\alpha_{n}\right)^{q-1}\right]=\infty$,

where $\mu=\max \left\{0,1-\left(\frac{q \lambda}{C_{q}}\right)^{\frac{1}{q-1}}\right\}, \lambda=\min _{j \in J}\left\{\lambda_{j}\right\}$, then the iterative sequence (5) converges weakly to a common fixed point of the family $\left\{T_{j}\right\}_{j=0}^{N-1}$.

Furthermore, we elicit a necessary and sufficient condition that guarantees strong convergence of the iterative sequence (5) to a common fixed point of the family $\left\{T_{j}\right\}_{j=0}^{N-1}$ in $q$-uniformly smooth Banach spaces.

We will use the notation:

1. $\rightarrow$ for weak convergence.

2. $\omega_{\mathcal{W}}\left(x_{n}\right)=\left\{x: \exists x_{n_{j}} \rightarrow x\right\}$ denotes the weak $\omega$-limit set of $\left\{x_{n}\right\}$.

\section{Preliminaries}

Let $E$ be a real Banach space. The modulus of smoothness of $E$ is the function $\rho_{E}:[0, \infty) \rightarrow$ $[0, \infty)$ defined by

$$
\rho_{E}(\tau)=\sup \left\{\frac{1}{2}(\|x+y\|+\|x-y\|)-1:\|x\| \leq 1,\|y\| \leq \tau\right\} .
$$

$E$ is uniformly smooth if and only if $\lim _{\tau \rightarrow 0}\left[\rho_{E}(\tau) / \tau\right]=0$.

Let $q>1$. $E$ is said to be $q$-uniformly smooth (or to have a modulus of smoothness of power type $q>1$ ) if there exists a constant $c>0$ such that $\rho_{E}(\tau) \leq c \tau^{q}$. Hilbert spaces, $L_{p}$ (or $\left.l_{p}\right)$ spaces $(1<p<\infty)$ and the Sobolev spaces $W_{m}^{p}(1<p<\infty)$ are $q$-uniformly smooth. Hilbert spaces are 2-uniformly smooth while

$$
L_{p}\left(\text { or } l_{p}\right) \quad \text { or } \quad W_{m}^{p} \quad \text { is } \begin{cases}p \text {-uniformly smooth } & \text { if } 1<p \leq 2 \\ 2 \text {-uniformly smooth } & \text { if } p \geq 2\end{cases}
$$

Theorem HKX ([9, p.1130]) Let $q>1$ and let E be a real q-uniformly smooth Banach space. Then there exists a constant $C_{q}>0$ such that, for all $x, y \in E$,

$$
\left.\|x+y\|^{q} \leq\|x\|^{q}+q \mid y, j_{q}(x)\right)+C_{q}\|y\|^{q} .
$$

$E$ is said to have a Fréchet differentiable norm if, for all $x \in U=\{x \in E:\|x\|=1\}$,

$$
\lim _{t \rightarrow 0} \frac{\|x+t y\|-\|x\|}{t}
$$

exists and is attained uniformly in $y \in U$. In this case, there exists an increasing function $b:[0, \infty) \rightarrow[0, \infty)$ with $\lim _{t \rightarrow 0^{+}}[b(t) / t]=0$ such that, for all $x, h \in E$,

$$
\frac{1}{2}\|x\|^{2}+\langle h, j(x)\rangle \leq \frac{1}{2}\|x+h\|^{2} \leq \frac{1}{2}\|x\|^{2}+\langle h, j(x)\rangle+b(\|h\|) .
$$


It is well known (see, for example, [10, p.107]) that a $q$-uniformly smooth Banach space has a Fréchet differentiable norm.

Lemma 2.1 ([5, p.1338]) Let E be a real q-uniformly smooth Banach space which is also uniformly convex. Let $K$ be a nonempty, closed and convex subset of $E$ and $T: K \rightarrow K$ be an asymptotically $\kappa$-strictly pseudocontractive mapping with a nonempty fixed point set. Then $(I-T)$ is demiclosed at zero, that is, if whenever $\left\{x_{n}\right\} \subset D(T)$ such that $\left\{x_{n}\right\}$ converges weakly to $x \in D(T)$ and $\left\{(I-T) x_{n}\right\}$ converges strongly to 0 , then $T x=x$.

Lemma 2.2 ([2, p.80]) Let $\left\{a_{n}\right\}_{n=0}^{\infty},\left\{b_{n}\right\}_{n=0}^{\infty},\left\{\delta_{n}\right\}_{n=0}^{\infty}$ be sequences of nonnegative real numbers satisfying the following inequality:

$$
a_{n+1} \leq\left(1+\delta_{n}\right) a_{n}+b_{n}, \quad \forall n \geq 0 .
$$

If $\sum_{n=0}^{\infty} \delta_{n}<\infty$ and $\sum_{n=0}^{\infty} b_{n}<\infty$, then $\lim _{n \rightarrow \infty} a_{n}$ exists. If, in addition, $\left\{a_{n}\right\}_{n=0}^{\infty}$ has a subsequence which converges strongly to zero, then $\lim _{n \rightarrow \infty} a_{n}=0$.

Lemma 2.3 ([2, p.78]) Let $E$ be a real Banach space, $K$ be a nonempty subset of $E$ and $T: K \rightarrow K$ be an asymptotically $\kappa$-strictly pseudocontractive mapping. Then $T$ is uniformly L-Lipschitzian.

Lemma 2.4 Let E be a real q-uniformly smooth Banach space which is also uniformly convex, and let $K$ be a nonempty, closed and convex subset of $E$. Let, for each $0 \leq j \leq N-1$, $T_{j}: K \rightarrow K$ be an asymptotically $\lambda_{j}$-strictly pseudocontractive mapping with $F \neq \emptyset$. Let $\left\{x_{n}\right\}_{n=0}^{\infty}$ be the sequence satisfying the following conditions:

(a) $\lim _{n \rightarrow \infty}\left\|x_{n}-p\right\|$ exists for every $p \in F$;

(b) $\lim _{n \rightarrow \infty}\left\|x_{n}-T_{j} x_{n}\right\|=0$, for each $0 \leq j \leq N-1$;

(c) $\lim _{n \rightarrow \infty}\left\|t x_{n}+(1-t) p_{1}-p_{2}\right\|$ exists for all $t \in[0,1]$ and for all $p_{1}, p_{2} \in F$.

Then the sequence $\left\{x_{n}\right\}$ converges weakly to a common fixed point of the family $\left\{T_{j}\right\}_{j=0}^{N-1}$.

Proof Since $\lim _{n \rightarrow \infty}\left\|x_{n}-p\right\|$ exists, then $\left\{x_{n}\right\}$ is bounded. By (b) and Lemma 2.1, we have $\omega_{\mathcal{W}}\left(x_{n}\right) \subset F$. Assume that $p_{1}, p_{2} \in \omega_{\mathcal{W}}\left(x_{n}\right)$ and that $\left\{x_{n_{i}}\right\}$ and $\left\{x_{m_{j}}\right\}$ are subsequences of $\left\{x_{n}\right\}$ such that $x_{n_{i}} \rightarrow p_{1}$ and $x_{m_{j}} \rightarrow p_{2}$, respectively. Since $E$ is a real $q$-uniformly smooth Banach space, which is also uniformly convex, then $E$ has a Fréchet differentiable norm. Set $x=p_{1}-p_{2}, h=t\left(x_{n}-p_{1}\right)$ in (8), we obtain

$$
\begin{aligned}
\frac{1}{2}\left\|p_{1}-p_{2}\right\|^{2}+t\left\langle x_{n}-p_{1}, j\left(p_{1}-p_{2}\right)\right\rangle \leq & \frac{1}{2}\left\|t x_{n}+(1-t) p_{1}-p_{2}\right\|^{2} \\
\leq & \frac{1}{2}\left\|p_{1}-p_{2}\right\|^{2}+b\left(t\left\|x_{n}-p_{1}\right\|\right) \\
& +t\left\langle x_{n}-p_{1}, j\left(p_{1}-p_{2}\right)\right\rangle,
\end{aligned}
$$

where $b$ is an increasing function. Since $\left\|x_{n}-p_{1}\right\| \leq M, \forall n \geq 0$, for some $M>0$, then

$$
\begin{aligned}
\frac{1}{2}\left\|p_{1}-p_{2}\right\|^{2}+t\left|x_{n}-p_{1}, j\left(p_{1}-p_{2}\right)\right\rangle & \leq \frac{1}{2}\left\|t x_{n}+(1-t) p_{1}-p_{2}\right\|^{2} \\
& \leq \frac{1}{2}\left\|p_{1}-p_{2}\right\|^{2}+b(t M)+t\left\langle x_{n}-p_{1}, j\left(p_{1}-p_{2}\right)\right\rangle .
\end{aligned}
$$


Therefore,

$$
\begin{aligned}
\frac{1}{2}\left\|p_{1}-p_{2}\right\|^{2}+t \limsup _{n \rightarrow \infty}\left\langle x_{n}-p_{1}, j\left(p_{1}-p_{2}\right)\right\rangle \leq & \frac{1}{2} \lim _{n \rightarrow \infty}\left\|t x_{n}+(1-t) p_{1}-p_{2}\right\|^{2} \\
\leq & \frac{1}{2}\left\|p_{1}-p_{2}\right\|^{2}+b(t M) \\
& +t \liminf _{n \rightarrow \infty}\left(x_{n}-p_{1}, j\left(p_{1}-p_{2}\right)\right\rangle .
\end{aligned}
$$

Hence, $\limsup _{n \rightarrow \infty}\left\langle x_{n}-p_{1}, j\left(p_{1}-p_{2}\right)\right\rangle \leq \liminf _{n \rightarrow \infty}\left\langle x_{n}-p_{1}, j\left(p_{1}-p_{2}\right)\right\rangle+b(t M) / t$. Since $\lim _{t \rightarrow 0^{+}}[b(t M) / t]=0$, then $\lim _{n \rightarrow \infty}\left\langle x_{n}-p_{1}, j\left(p_{1}-p_{2}\right)\right\rangle$ exists. Since $\lim _{n \rightarrow \infty}\left\langle x_{n}-p_{1}, j\left(p_{1}-\right.\right.$ $\left.\left.p_{2}\right)\right\rangle=\left\langle p-p_{1}, j\left(p_{1}-p_{2}\right)\right\rangle$, for all $p \in \omega_{\mathcal{W}}\left(x_{n}\right)$. Set $p=p_{2}$. We have $\left\langle p_{2}-p_{1}, j\left(p_{1}-p_{2}\right)\right\rangle=0$, that is, $p_{2}=p_{1}$. Hence, $\omega_{\mathcal{W}}\left(x_{n}\right)$ is a singleton, so that $\left\{x_{n}\right\}$ converges weakly to a common fixed point of the family $\left\{T_{j}\right\}_{j=0}^{N-1}$.

\section{Main results}

Theorem 3.1 Let E be a real q-uniformly smooth Banach space which is also uniformly convex and $K$ be a nonempty, closed and convex subset of $E$. Let $N \geq 1$ be an integer and $J=\{0,1,2, \ldots, N-1\}$. Let, for each $j \in J, T_{j}: K \rightarrow K$ be an asymptotically $\lambda_{j}$-strictly pseudocontractive mapping for some $0 \leq \lambda_{j}<1$ with sequences $\left\{\kappa_{n, j}\right\}_{n=0}^{\infty} \subset[1, \infty)$ such that $\sum_{n=0}^{\infty}\left(\kappa_{n}-1\right)<\infty$, where $\kappa_{n}=\max _{j \in J}\left\{\kappa_{n, j}\right\}$, and $F:=\bigcap_{j=0}^{N-1} F\left(T_{j}\right) \neq \emptyset$. Let $\lambda=\min _{j \in J}\left\{\lambda_{j}\right\}$. Let $\left\{\alpha_{n}\right\}$ satisfy the conditions (6) and $\left\{x_{n}\right\}$ be the sequence generated by the cyclic algorithm (5). Then $\left\{x_{n}\right\}$ converges weakly to a common fixed point of the family $\left\{T_{j}\right\}_{j=0}^{N-1}$.

Proof Pick a $p \in F$. We firstly show that $\lim _{n \rightarrow \infty}\left\|x_{n}-p\right\|$ exists. To see this, using (2) and (7), we obtain

$$
\begin{aligned}
\left\|x_{n+1}-p\right\|^{q}= & \left\|x_{n}-p-\left(1-\alpha_{n}\right)\left[x_{n}-p-\left(T_{i(n)}^{k(n)} x_{n}-p\right)\right]\right\|^{q} \\
\leq & \left\|x_{n}-p\right\|^{q}+C_{q}\left(1-\alpha_{n}\right)^{q}\left\|x_{n}-p-\left(T_{i(n)}^{k(n)} x_{n}-p\right)\right\|^{q} \\
& -q\left(1-\alpha_{n}\right)\left\{x_{n}-p-\left(T_{i(n)}^{k(n)} x_{n}-p\right), j_{q}\left(x_{n}-p\right)\right\rangle \\
\leq & \left\|x_{n}-p\right\|^{q}+C_{q}\left(1-\alpha_{n}\right)^{q}\left\|x_{n}-p-\left(T_{i(n)}^{k(n)} x_{n}-p\right)\right\|^{q} \\
& -q\left(1-\alpha_{n}\right)\left\{\lambda_{i(n)}\left\|x_{n}-p-\left(T_{i(n)}^{k(n)} x_{n}-p\right)\right\|^{q}\right. \\
& \left.-\left(\kappa_{k(n), i(n)}-1\right)\left\|x_{n}-p\right\|^{q}\right\} \\
= & {\left[1+q\left(1-\alpha_{n}\right)\left(\kappa_{k(n), i(n)}-1\right)\right]\left\|x_{n}-p\right\|^{q} } \\
& -\left(1-\alpha_{n}\right)\left[q \lambda_{i(n)}-C_{q}\left(1-\alpha_{n}\right)^{q-1}\right]\left\|x_{n}-T_{i(n)}^{k(n)} x_{n}\right\|^{q} \\
\leq & {\left[1+q\left(1-\alpha_{n}\right)\left(\kappa_{k(n)}-1\right)\right]\left\|x_{n}-p\right\|^{q} } \\
& -\left(1-\alpha_{n}\right)\left[q \lambda-C_{q}\left(1-\alpha_{n}\right)^{q-1}\right]\left\|x_{n}-T_{i(n)}^{k(n)} x_{n}\right\|^{q},
\end{aligned}
$$

where $\kappa_{k(n)}=\max _{i \in J}\left\{\kappa_{k(n), i(n)}\right\}$. Since $\mu \leq \alpha_{n}<1$ for all $n$, where $\mu=\max \left\{0,1-\left(\frac{q \lambda}{C_{q}}\right)^{\frac{1}{q-1}}\right\}$, we get $\left(1-\alpha_{n}\right)\left[q \lambda-C_{q}\left(1-\alpha_{n}\right)^{q-1}\right] \geq 0$. Therefore, (9) implies

$$
\left\|x_{n+1}-p\right\|^{q} \leq\left[1+q\left(1-\alpha_{n}\right)\left(\kappa_{k(n)}-1\right)\right]\left\|x_{n}-p\right\|^{q} .
$$


Let $\delta_{n}=1+q\left(1-\alpha_{n}\right)\left(\kappa_{k(n)}-1\right)$. Since $\sum_{n=0}^{\infty}\left(\kappa_{n}-1\right)<\infty$, we have

$$
\sum_{n=0}^{\infty}\left(\delta_{n}-1\right)=q \sum_{n=0}^{\infty}\left(1-\alpha_{n}\right)\left(\kappa_{k(n)}-1\right) \leq q N \sum_{n=1}^{\infty}\left(\kappa_{n}-1\right)<\infty,
$$

then (10) implies $\lim _{n \rightarrow \infty}\left\|x_{n}-p\right\|$ exists by Lemma 2.2 (and hence the sequence $\left\{\left\|x_{n}-p\right\|\right\}$ is bounded, that is, there exists a constant $M>0$ such that $\left.\left\|x_{n}-p\right\|<M\right)$.

Then we prove $\lim _{n \rightarrow \infty}\left\|x_{n}-T_{j} x_{n}\right\|=0, \forall j \in J$. In fact, it follows from (9) that

$$
\begin{aligned}
\left(1-\alpha_{n}\right)\left[q \lambda-C_{q}\left(1-\alpha_{n}\right)^{q-1}\right]\left\|x_{n}-T_{i(n)}^{k(n)} x_{n}\right\|^{q} \leq & \left\|x_{n}-p\right\|^{q}-\left\|x_{n+1}-p\right\|^{q} \\
& +q\left(1-\alpha_{n}\right)\left(\kappa_{k(n)}-1\right)\left\|x_{n}-p\right\|^{q} .
\end{aligned}
$$

Then

$$
\sum_{n=0}^{\infty}\left(1-\alpha_{n}\right)\left[q \lambda-C_{q}\left(1-\alpha_{n}\right)^{q-1}\right]\left\|x_{n}-T_{i(n)}^{k(n)} x_{n}\right\|^{q}<\left\|x_{0}-p\right\|^{q}+M^{q} \sum_{n=0}^{\infty}\left(\delta_{k(n)}-1\right)<\infty .
$$

Since $\sum_{n=0}^{\infty}\left(1-\alpha_{n}\right)\left[q \lambda-C_{q}\left(1-\alpha_{n}\right)^{q-1}\right]=\infty$, then (11) implies that $\liminf _{n \rightarrow \infty} \| x_{n}-$ $T_{i(n)}^{k(n)} x_{n} \|=0$. Thus $\lim _{n \rightarrow \infty}\left\|x_{n}-T_{i(n)}^{k(n)} x_{n}\right\|=0$.

For all $n>N$, we have $k(n)-1=k(n-N)$ and $i(n)=i(n-N)$. By Lemma 2.3, we know that $T_{j}$ is uniformly $L_{j}$-Lipschitzian, then there exists a constant $L=\max _{j \in J}\left\{L_{j}\right\}$, such that

$$
\left\|T_{j}^{n} x-T_{j}^{n} y\right\| \leq L\|x-y\|, \quad \forall n \geq 0, \forall x, y \in K \text { and } \forall j \in J .
$$

Thus

$$
\begin{aligned}
\left\|x_{n}-T_{i(n)} x_{n}\right\| \leq & \left\|x_{n}-T_{i(n)}^{k(n)} x_{n}\right\|+\left\|T_{i(n)}^{k(n)} x_{n}-T_{i(n)} x_{n}\right\| \\
\leq & \left\|x_{n}-T_{i(n)}^{k(n)} x_{n}\right\|+L\left\|T_{i(n)}^{k(n)-1} x_{n}-x_{n}\right\| \\
\leq & \left\|x_{n}-T_{i(n)}^{k(n)} x_{n}\right\|+L\left\|T_{i(n)}^{k(n)-1} x_{n}-T_{i(n-N)}^{k(n)-1} x_{n-N}\right\| \\
& \quad+L\left\|T_{i(n-N)}^{k(n)-1} x_{n-N}-x_{n-N-1}\right\|+L\left\|x_{n-N-1}-x_{n}\right\| \\
\leq & \left\|x_{n}-T_{i(n)}^{k(n)} x_{n}\right\|+L^{2}\left\|x_{n}-x_{n-N}\right\| \\
& \quad+L\left\|T_{i(n-N)}^{k(n-N)} x_{n-N}-x_{n-N-1}\right\|+L\left\|x_{n-N-1}-x_{n}\right\| .
\end{aligned}
$$

Observe that

$$
\left\|x_{n}-x_{n+1}\right\|=\left(1-\alpha_{n}\right)\left\|x_{n}-T_{i(n)}^{k(n)} x_{n}\right\| \rightarrow 0 \quad \text { as } n \rightarrow \infty .
$$

Consequently,

$$
\left\|x_{n}-x_{n+l}\right\| \rightarrow 0 \quad \text { as } n \rightarrow \infty \text {, for all integer } l
$$

Observe also that

$$
\left\|x_{n-1}-T_{i(n)}^{k(n)} x_{n}\right\| \leq\left\|x_{n}-x_{n-1}\right\|+\left\|x_{n}-T_{i(n)}^{k(n)} x_{n}\right\| \rightarrow 0 \quad \text { as } n \rightarrow \infty .
$$


Hence,

$$
\lim _{n \rightarrow \infty}\left\|x_{n}-T_{i(n)} x_{n}\right\|=0
$$

Consequently, for all $j \in J$, we have

$$
\left\|x_{n}-T_{n+j} x_{n}\right\| \leq\left\|x_{n}-x_{n+j}\right\|+\left\|x_{n+j}-T_{n+j} x_{n+j}\right\|+L\left\|x_{n}-x_{n+j}\right\| \rightarrow 0 \quad \text { as } n \rightarrow \infty
$$

Thus,

$$
\lim _{n \rightarrow \infty}\left\|x_{n}-T_{j} x_{n}\right\|=0, \quad \forall j \in J .
$$

Now we prove that for all $p_{1}, p_{2} \in F, \lim _{n \rightarrow \infty}\left\|t x_{n}+(1-t) p_{1}-p_{2}\right\|$ exists for all $t \in$ $[0,1]$. Let $a_{n}(t)=\left\|t x_{n}+(1-t) p_{1}-p_{2}\right\|$. It is obvious that $\lim _{n \rightarrow \infty} a_{n}(0)=\left\|p_{1}-p_{2}\right\|$ and $\lim _{n \rightarrow \infty} a_{n}(1)=\lim _{n \rightarrow \infty}\left\|x_{n}-p_{2}\right\|$ exist. So, we only need to consider the case of $t \in(0,1)$. Define $A_{n}: K \rightarrow K$ by

$$
A_{n} x=\alpha_{n} x+\left(1-\alpha_{n}\right) T_{i(n)}^{k(n)} x, \quad x \in K
$$

Then for all $x, y \in K$

$$
\begin{aligned}
\left\|A_{n} x-A_{n} y\right\|^{q} \leq & \|x-y\|^{q}-q\left(1-\alpha_{n}\right)\left\langle\left(I-T_{i(n)}^{k(n)}\right) x-\left(I-T_{i(n)}^{k(n)}\right) y, j_{q}(x-y)\right\rangle \\
& +C_{q}\left(1-\alpha_{n}\right)^{q}\left\|x-y-\left(T_{i(n)}^{k(n)} x-T_{i(n)}^{k(n)} y\right)\right\|^{q} \\
\leq & {\left[1+q\left(1-\alpha_{n}\right)\left(\kappa_{k(n)}-1\right)\right]\|x-y\|^{q} } \\
& -\left(1-\alpha_{n}\right)\left[q \lambda-C_{q}\left(1-\alpha_{n}\right)^{q-1}\right]\left\|x-y-\left(T_{i(n)}^{k(n)} x-T_{i(n)}^{k(n)} y\right)\right\|^{q} .
\end{aligned}
$$

By the choice of $\alpha_{n}$, we have $\left(1-\alpha_{n}\right)\left[q \lambda-C_{q}\left(1-\alpha_{n}\right)^{q-1}\right] \geq 0$, so it follows that $\| A_{n} x-$ $A_{n} y\left\|^{q} \leq\left[1+q\left(1-\alpha_{n}\right)\left(\kappa_{k(n)}-1\right)\right]\right\| x-y\left\|^{q}=\delta_{n}\right\| x-y \|^{q}$. For the convenience of the following discussion, set $\eta_{n}=\left(\delta_{n}\right)^{\frac{1}{q}}$, then $\left\|A_{n} x-A_{n} y\right\| \leq \eta_{n}\|x-y\|$.

Set $S_{n, m}=A_{n+m-1} A_{n+m-2} \cdots A_{n}, m \geq 1$. We have

$$
\left\|S_{n, m} x-S_{n, m} y\right\| \leq\left(\prod_{j=n}^{n+m-1} \eta_{j}\right)\|x-y\| \quad \text { for all } x, y \in K,
$$

and

$$
S_{n, m} x_{n}=x_{n+m}, \quad S_{n, m} p=p \quad \text { for all } p \in F .
$$

Set $b_{n, m}=\left\|S_{n, m}\left(t x_{n}+(1-t) p_{1}\right)-t S_{n, m} x_{n}-(1-t) S_{n, m} p_{1}\right\|$. If $\left\|x_{n}-p_{1}\right\|=0$ for some $n_{0}$, then $x_{n}=p_{1}$ for any $n \geq n_{0}$ so that $\lim _{n \rightarrow \infty}\left\|x_{n}-p_{1}\right\|=0$, in fact, $\left\{x_{n}\right\}$ converges strongly to $p_{1} \in F$. Thus we may assume $\left\|x_{n}-p_{1}\right\|>0$ for any $n \geq 0$. Let $\delta$ denote the modulus of convexity of $E$. It is well known (see, for example, [11, p.108]) that

$$
\begin{aligned}
\|t x+(1-t) y\| & \leq 1-2 \min \{t,(1-t)\} \delta(\|x-y\|) \\
& \leq 1-2 t(1-t) \delta(\|x-y\|)
\end{aligned}
$$


for all $t \in[0,1]$ and for all $x, y \in E$ such that $\|x\| \leq 1,\|y\| \leq 1$. Set

$$
w_{n, m}=\frac{S_{n, m} p_{1}-S_{n, m}\left(t x_{n}+(1-t) p_{1}\right)}{t\left(\prod_{j=n}^{n+m-1} \eta_{j}\right)\left\|x_{n}-p_{1}\right\|}, \quad z_{n, m}=\frac{S_{n, m}\left(t x_{n}+(1-t) p_{1}\right)-S_{n, m} x_{n}}{(1-t)\left(\prod_{j=n}^{n+m-1} \eta_{j}\right)\left\|x_{n}-p_{1}\right\|} .
$$

Then $\left\|w_{n, m}\right\| \leq 1$ and $\left\|z_{n, m}\right\| \leq 1$ so that it follows from (12) that

$$
2 t(1-t) \delta\left(\left\|w_{n, m}-z_{n, m}\right\|\right) \leq 1-\left\|t w_{n, m}+(1-t) z_{n, m}\right\| .
$$

Observe that

$$
\left\|w_{n, m}-z_{n, m}\right\|=\frac{b_{n, m}}{t(1-t)\left(\prod_{j=n}^{n+m-1} \eta_{j}\right)\left\|x_{n}-p_{1}\right\|}
$$

and

$$
\left\|t w_{n, m}+(1-t) z_{n, m}\right\|=\frac{\left\|S_{n, m} x_{n}-S_{n, m} p_{1}\right\|}{\left(\prod_{j=n}^{n+m-1} \eta_{j}\right)\left\|x_{n}-p_{1}\right\|},
$$

it follows from (13) that

$$
\begin{aligned}
2 t & (1-t)\left(\prod_{j=n}^{n+m-1} \eta_{j}\right)\left\|x_{n}-p_{1}\right\| \delta\left(\frac{b_{n, m}}{t(1-t)\left(\prod_{j=n}^{n+m-1} \eta_{j}\right)\left\|x_{n}-p_{1}\right\|}\right) \\
& \leq\left(\prod_{j=n}^{n+m-1} \eta_{j}\right)\left\|x_{n}-p_{1}\right\|-\left\|S_{n, m} x_{n}-S_{n, m} p_{1}\right\| \\
& =\left(\prod_{j=n}^{n+m-1} \eta_{j}\right)\left\|x_{n}-p_{1}\right\|-\left\|x_{n+m}-p_{1}\right\| .
\end{aligned}
$$

Since $E$ is uniformly convex, then $\delta(s) / s$ is nondecreasing, and since $\left(\prod_{j=n}^{n+m-1} \eta_{j}\right) \| x_{n}-$ $p_{1}\left\|\leq\left(\prod_{j=n}^{n+m-1} \eta_{j}\right) \eta_{n-1}\right\| x_{n-1}-p_{1}\left\|\leq \cdots \leq\left(\prod_{j=n}^{n+m-1} \eta_{j}\right)\left(\prod_{j=0}^{n-1} \eta_{j}\right)\right\| x_{0}-p_{1}\left\|=\left(\prod_{j=0}^{n+m-1} \eta_{j}\right)\right\| x_{0}-$ $p_{1} \|$, hence it follows from (14) that

$$
\begin{aligned}
& \frac{\left(\prod_{j=0}^{n+m-1} \eta_{j}\right)\left\|x_{0}-p_{1}\right\|}{2} \delta\left(\frac{4}{\left(\prod_{j=0}^{n+m-1} \eta_{j}\right)\left\|x_{0}-p_{1}\right\|} b_{n, m}\right) \\
& \quad \leq\left(\prod_{j=n}^{n+m-1} \eta_{j}\right)\left\|x_{n}-p_{1}\right\|-\left\|x_{n+m}-p_{1}\right\| \quad\left(\text { since } t(1-t) \leq \frac{1}{4} \text { for all } t \in[0,1]\right) .
\end{aligned}
$$

Since $\lim _{n \rightarrow \infty} \prod_{j=0}^{n+m-1} \eta_{j}$ exits and $\lim _{n \rightarrow \infty} \prod_{j=0}^{n+m-1} \eta_{j} \neq 0$. Also since $\lim _{n \rightarrow \infty} \prod_{j=n}^{n+m-1} \eta_{j}=1$ and $\lim _{n \rightarrow \infty}\left\|x_{n}-p_{1}\right\|$ exists, then the continuity of $\delta$ and $\delta(0)=0$ yield $\lim _{n \rightarrow \infty} b_{n, m}=0$ uniformly for all $m \geq 1$. Observe that

$$
\begin{aligned}
a_{n+m}(t) \leq & \| t x_{n+m}+(1-t) p_{1}-p_{2}+\left(S_{n, m}\left(t x_{n}+(1-t) p_{1}\right)\right. \\
& \left.-t S_{n, m} x_{n}-(1-t) S_{n, m} p_{1}\right) \| \\
& +\left\|S_{n, m}\left(t x_{n}+(1-t) p_{1}\right)-t S_{n, m} x_{n}-(1-t) S_{n, m} p_{1}\right\|
\end{aligned}
$$




$$
\begin{aligned}
& =\left\|S_{n, m}\left(t x_{n}+(1-t) p_{1}\right)-S_{n, m} p_{2}\right\|+b_{n, m} \\
& \leq\left(\prod_{j=n}^{n+m-1} \eta_{j}\right)\left\|t x_{n}+(1-t) p_{1}-p_{2}\right\|+b_{n, m}=\left(\prod_{j=n}^{n+m-1} \eta_{j}\right) a_{n}(t)+b_{n, m} .
\end{aligned}
$$

Hence $\limsup _{n \rightarrow \infty} a_{n}(t) \leq \liminf _{n \rightarrow \infty} a_{n}(t)$, this ensures that $\lim _{n \rightarrow \infty} a_{n}(t)$ exists for all $t \in(0,1)$.

Now apply Lemma 2.4 to conclude that $\left\{x_{n}\right\}$ converges weakly to a common fixed point of the family $\left\{T_{j}\right\}_{j=0}^{N-1}$.

Theorem 3.2 Let E be a real q-uniformly smooth Banach space, and let $K$ be a nonempty, closed and convex subset of $E$. Let $N \geq 1$ be an integer and $J=\{0,1,2, \ldots, N-1\}$. Let, for each $j \in J, T_{j}: K \rightarrow K$ be an asymptotically $\lambda_{j}$-strictly pseudocontractive mapping for some $0 \leq$ $\lambda_{j}<1$ with sequences $\left\{\kappa_{n, j}\right\}_{n=0}^{\infty} \subset[1, \infty)$ such that $\sum_{n=0}^{\infty}\left(\kappa_{n}-1\right)<\infty$, where $\kappa_{n}=\max _{j \in J}\left\{\kappa_{n, j}\right\}$, and $F:=\bigcap_{j=0}^{N-1} F\left(T_{j}\right) \neq \emptyset$. Let $\lambda=\min _{j \in J}\left\{\lambda_{j}\right\}$. Let $\left\{\alpha_{n}\right\}$ satisfy the conditions (6) and $\left\{x_{n}\right\}$ be the sequence generated by the cyclic algorithm (5). Then $\left\{x_{n}\right\}$ converges strongly to a common fixed point of the family $\left\{T_{j}\right\}_{j=0}^{N-1}$ if and only if

$$
\liminf _{n \rightarrow \infty} d\left(x_{n}, F\right)=0
$$

where $d\left(x_{n}, F\right)=\inf _{p \in F}\left\|x_{n}-p\right\|$.

Proof It follows from (10) that

$$
\left\|x_{n+1}-p\right\|^{q} \leq \delta_{n}\left\|x_{n}-p\right\|^{q}
$$

Thus $\left[d\left(x_{n+1}-p\right)\right]^{q} \leq \delta_{n}\left[d\left(x_{n}-p\right)\right]^{q}$, and it follows from Lemma 2.2 that $\lim _{n \rightarrow \infty} d\left(x_{n}, F\right)$ exists.

Now if $\left\{x_{n}\right\}$ converges strongly to a common fixed point $p$ of the family $\left\{T_{j}\right\}_{j=0}^{N-1}$, then $\lim _{n \rightarrow \infty}\left\|x_{n}-p\right\|=0$. Since

$$
0 \leq d\left(x_{n}, F\right) \leq\left\|x_{n}-p\right\|
$$

we have $\liminf _{n \rightarrow \infty} d\left(x_{n}, F\right)=0$.

Conversely, suppose $\liminf _{n \rightarrow \infty} d\left(x_{n}, F\right)=0$, then the existence of $\lim _{n \rightarrow \infty} d\left(x_{n}, F\right)$ implies that $\lim _{n \rightarrow \infty} d\left(x_{n}, F\right)=0$. Thus, for arbitrary $\epsilon>0$, there exists a positive integer $n_{0}$ such that $d\left(x_{n}, F\right)<\frac{\epsilon}{2}$ for any $n \geq n_{0}$.

From (10), we have

$$
\left\|x_{n+1}-p\right\|^{q} \leq\left\|x_{n}-p\right\|^{q}+M^{q}\left(\delta_{n}-1\right), \quad n \geq 0,
$$

and for some $M>0,\left\|x_{n}-p\right\|<M$. Now, an induction yields

$$
\begin{aligned}
\left\|x_{n}-p\right\|^{q} & \leq\left\|x_{n-1}-p\right\|^{q}+M^{q}\left(\delta_{n-1}-1\right) \\
& \leq\left\|x_{n-2}-p\right\|^{q}+M^{q}\left(\delta_{n-2}-1\right)+M^{q}\left(\delta_{n-1}-1\right) \\
& \leq \cdots \leq\left\|x_{l}-p\right\|^{q}+M^{q} \sum_{j=l}^{n-1}\left(\delta_{j}-1\right), \quad n-1 \geq l \geq 0 .
\end{aligned}
$$


Since $\sum_{n=0}^{\infty}\left(\delta_{n}-1\right)<\infty$, then there exists a positive integer $n_{1}$ such that $\sum_{j=n}^{\infty}\left(\delta_{j}-1\right)<\left(\frac{\epsilon}{2 M}\right)^{q}$, $\forall n \geq n_{1}$. Choose $N=\max \left\{n_{0}, n_{1}\right\}$, then for all $n, m \geq N+1$ and for all $p \in F$, we have

$$
\begin{aligned}
\left\|x_{n}-x_{m}\right\| & \leq\left\|x_{n}-p\right\|+\left\|x_{m}-p\right\| \\
& \leq\left[\left\|x_{N}-p\right\|^{q}+M^{q} \sum_{j=N}^{n-1}\left(\delta_{j}-1\right)\right]^{\frac{1}{q}}+\left[\left\|x_{N}-p\right\|^{q}+M^{q} \sum_{j=N}^{m-1}\left(\delta_{j}-1\right)\right]^{\frac{1}{q}} \\
& \leq\left[\left\|x_{N}-p\right\|^{q}+M^{q} \sum_{j=N}^{\infty}\left(\delta_{j}-1\right)\right]^{\frac{1}{q}}+\left[\left\|x_{N}-p\right\|^{q}+M^{q} \sum_{j=N}^{\infty}\left(\delta_{j}-1\right)\right]^{\frac{1}{q}} \\
& =2\left[\left\|x_{N}-p\right\|^{q}+M^{q} \sum_{j=N}^{\infty}\left(\delta_{j}-1\right)\right]^{\frac{1}{q}} .
\end{aligned}
$$

Taking infimum over all $p \in F$, we obtain

$$
\begin{aligned}
\left\|x_{n}-x_{m}\right\| & \leq 2\left\{\left[d\left(x_{N}, F\right)\right]^{q}+M^{q} \sum_{j=N}^{\infty}\left(\delta_{j}-1\right)\right\}^{\frac{1}{q}} \\
& <2\left[\left(\frac{\epsilon}{2}\right)^{q}+M^{q}\left(\frac{\epsilon}{2 M}\right)^{q}\right]^{\frac{1}{q}}<2 \epsilon .
\end{aligned}
$$

Thus $\left\{x_{n}\right\}_{n=0}^{\infty}$ is Cauchy. Suppose $\lim _{n \rightarrow x_{n}}=u$. Then for all $j \in J$ we have

$$
0 \leq\left\|u-T_{j} u\right\| \leq\left\|u-x_{n}\right\|+\left\|x_{n}-T_{j} x_{n}\right\|+L\left\|x_{n}-u\right\| \rightarrow 0 \quad \text { as } n \rightarrow \infty .
$$

Thus $u \in F\left(T_{j}\right), \forall j \in J$, and hence $u \in F$.

\section{Competing interests}

The authors declare that they have no competing interests.

\section{Authors' contributions}

All the authors have read and approved the final manuscript.

\section{Author details}

${ }^{1}$ School of Mathematics and Physics, North China Electric Power University, Baoding, Hebei 071003, P.R. China. ${ }^{2}$ School of Economics, Renmin University of China, Beijing, 100872, P.R. China. ${ }^{3}$ Easyway Company Limited, Beijing, 100872, P.R. China.

Received: 9 June 2012 Accepted: 13 September 2012 Published: 2 October 2012

\section{References}

1. Osilike, MO: Iterative approximations of fixed points of asymptotically demicontractive mappings. Indian J. Pure Appl. Math. 29(12), 1291-1300 (1998)

2. Osilike, MO, Aniagbosor, SC, Akuchu, BG: Fixed points of asymptotically demicontractive mappings in arbitrary Banach spaces. Panam. Math. J. 12(2), 77-88 (2002)

3. Qihou, L: Convergence theorems of the sequence of iterates for asymptotically demicontractive and hemicontractive mappings. Nonlinear Anal. 26(11), 1835-1842 (1996)

4. Browder, FE, Petryshyn, WV: Construction of fixed points of nonlinear mappings in Hilbert space. J. Math. Anal. Appl. 20, 197-228 (1967)

5. Osilike, MO, Udomene, A, lgbokwe, DI, Akuchu, BG: Demiclosedness principle and convergence theorems for $\kappa$-strictly asymptotically pseudo-contractive maps. J. Math. Anal. Appl. 326, 1334-1345 (2007)

6. Acedo, GL, Xu, HK: Iterative methods for strict pseudo-contractions in Hilbert spaces. Nonlinear Anal. 67, 2258-2271 (2007)

7. Qin, $X, C h o, Y J, K u, S M$, Shang, M: A hybrid iterative scheme for asymptotically $k$-strict pseudo-contractions in Hilbert spaces. Nonlinear Anal. 70, 1902-1911 (2009) 
8. Osilike, MO, Shehu, Y: Explicit averaging cyclic algorithm for common fixed points of a finite family of asymptotically strictly pseudocontractive maps in Banach spaces. Comput. Math. Appl. 57, 1502-1510 (2009)

9. Xu, HK: Inequalities in Banach spaces with applications. Nonlinear Anal. 16, 1127-1138 (1991)

10. Takahashi, W: Nonlinear Functional Analysis. Fixed Point Theory and Its Applications. Yokohama, Yokohama (2000)

11. Bruck, RE: A simple proof of the mean ergodic theorem for nonlinear contractions in Banach spaces. Isr. J. Math. 32(2-3), 107-116 (1979)

doi:10.1186/1687-1812-2012-167

Cite this article as: Zhang and Xie: Explicit averaging cyclic algorithm for common fixed points of a finite family of asymptotically strictly pseudocontractive mappings in q-uniformly smooth Banach spaces. Fixed Point Theory and Applications 2012 2012:167.

Submit your manuscript to a SpringerOpen ${ }^{\odot}$ journal and benefit from:

- Convenient online submission

- Rigorous peer review

- Immediate publication on acceptance

- Open access: articles freely available online

- High visibility within the field

- Retaining the copyright to your article

Submit your next manuscript at $>$ springeropen.com 